\title{
Best Approximation of Ruspini Partitions in Gödel Logic
}

\author{
Pietro Codara ${ }^{1}$, Ottavio M. D'Antona ${ }^{2}$, and Vincenzo Marra ${ }^{2}$ \\ 1 Dipartimento di Matematica F. Enriques, Università degli Studi di Milano, \\ via Saldini 50, I-20133 Milano, Italy, \\ codara@mat.unimi.it, \\ 2 Dipartimento di Informatica e Comunicazione, Università degli Studi di Milano, \\ via Comelico 39, I-20135 Milano, Italy, \\ \{dantona,marra\}@dico.unimi.it
}

\begin{abstract}
A Ruspini partition is a finite family of fuzzy sets $\left\{f_{1}, \ldots, f_{n}\right\}$, $f_{i}:[0,1] \rightarrow[0,1]$, such that $\sum_{i=1}^{n} f_{i}(x)=1$ for all $x \in[0,1]$. We analyze such partitions in the language of Gödel logic. Our main result identifies the precise degree to which the Ruspini condition is expressible in this language, and yields inter alia a constructive procedure to axiomatize a given Ruspini partition by a theory in Gödel logic.
\end{abstract}

\section{Introduction}

Let $[0,1]$ be the real unit interval. By a fuzzy set we shall mean a function $f:[0,1] \rightarrow[0,1]$. Throughout the paper, we fix a finite nonempty family

$$
P=\left\{f_{1}, \ldots, f_{n}\right\}
$$

of fuzzy sets, for $n \geq 1$ an integer. Moreover, we write $\underline{n}$ for the set $\{1, \ldots, n\}$.

In several soft computing applications, the following notion of fuzzy partition plays an important role. It is often traced back to [9, p. 28].

Definition 1. We say $P$ is a Ruspini partition if for all $x \in[0,1]$

$$
\sum_{i=1}^{n} f_{i}(x)=1
$$

By way of informal motivation for what follows, think of the real unit interval $[0,1]$ as the normalized range of values of a physical observable, say "Temperature". Then each $f_{i} \in P$ can be viewed as a means of assigning a truth-value to a proposition about temperature in some many-valued logic $\mathscr{L}$. Had one no information at all about such propositions, one would be led to identify them with propositional variables $X_{i}$, subject only to the axioms of $\mathscr{L}$. However, the set $P$ does encode information about $X_{1}, \ldots, X_{n}$. For example, consider $P=\left\{f_{1}, f_{2}, f_{3}\right\}$ as in Fig. 1 , and say $f_{1}, f_{2}$, and $f_{3}$ provide truth-values for the propositions $X_{1}=$ "The temperature is low", $X_{2}=$ "The temperature is 

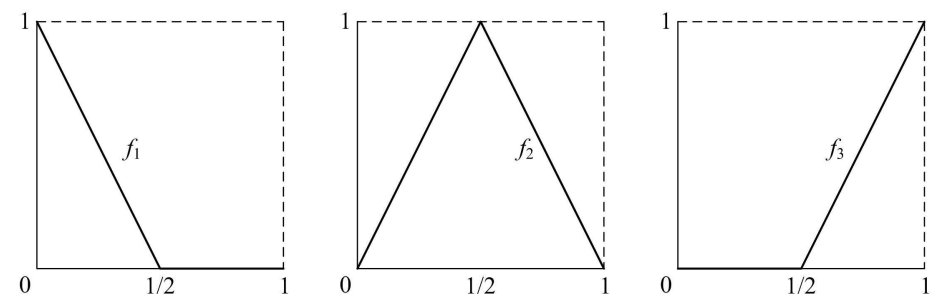

Fig. 1. A Ruspini partition $\left\{f_{1}, f_{2}, f_{3}\right\}$.

medium", and $X_{3}=$ "The temperature is high", respectively. If $\mathscr{L}$ has a conjunction $\wedge$ interpreted by minimum, the proposition $X_{1} \wedge X_{3}$ has 0 as its only possible truth-value, i.e., it is a contradiction. The set $P$ then leads one to add extra-logical axioms to $\mathscr{L}$, e.g. $\neg\left(X_{1} \wedge X_{3}\right)$, in an attempt to express the fact that one cannot observe both a high and a low temperature at the same time. More generally, $P$ implicitly encodes a theory over the pure logic $\mathscr{L}$.

Throughout the paper, we shall take $\mathscr{L}$ to be Gödel logic. Recall that Gödel (propositional) logic can be defined as the schematic extension of the intuitionistic propositional calculus by the prelinearity axiom $(\alpha \rightarrow \beta) \vee(\beta \rightarrow \alpha)$. It can also be semantically defined as a many-valued logic, as follows. Let us consider well-formed formulas over propositional variables $X_{1}, X_{2}, \ldots$ in the language $\wedge, \vee, \rightarrow, \neg, \perp, \top$. (We use $\perp$ and $\top$ as the logical constants falsum and verum, respectively). By an assignment we shall mean a function $\mu$ from (well-formed) formulas to $[0,1] \subseteq \mathbb{R}$ such that, for any two such formulas $\alpha, \beta$,

$$
\begin{aligned}
& \mu(\alpha \wedge \beta)=\min \{\mu(\alpha), \mu(\beta)\} \\
& \mu(\alpha \vee \beta)=\max \{\mu(\alpha), \mu(\beta)\} \\
& \mu(\alpha \rightarrow \beta)= \begin{cases}1 & \text { if } \mu(\alpha) \leq \mu(\beta) \\
\mu(\beta) & \text { otherwise }\end{cases}
\end{aligned}
$$

and $\mu(\neg \alpha)=\mu(\alpha \rightarrow \perp), \mu(\perp)=0, \mu(\top)=1$. A tautology is a formula $\alpha$ such that $\mu(\alpha)=1$ for every assignment $\mu$. As is well-known, Gödel logic is complete with respect to this many-valued semantics. We refer to e.g. [6] [7] for detailed treatments.

This paper provides a thorough analysis of how the Ruspini condition on $P$ is reflected by the resulting theory over Gödel logic. In our main Theorem, we shall eventually obtain a constructive procedure to axiomatize the theory implicitly encoded by $P$. While it is to be expected that Gödel logic cannot precisely capture addition of real numbers in the Ruspini condition (1), our main result proves that, up to logical equivalence, (1) reduces to the notion of weak Ruspini partition in Definition 7. In Section 2 we collect the necessary algebraic and combinatorial background, and prove some preliminary results. In Section 3 we establish our main result. The final Section 4 summarizes our findings. 


\section{Preliminary Results}

We make use of the algebraic counterpart of Gödel logic, namely, the variety of Gödel algebras. These are Heyting algebras $\langle G, \wedge, \vee, \neg, \top, \perp\rangle$ satisfying the prelinearity condition $(x \rightarrow y) \vee(y \rightarrow x)=\top$.

The collection of all functions from $[0,1]$ to $[0,1]$ has the structure of a Gödel algebra under the following operations, for $f, g:[0,1] \rightarrow[0,1]$.

$$
\begin{aligned}
& (f \wedge g)(x)=\min \{f(x), g(x)\} \\
& (f \vee g)(x)=\max \{f(x), g(x)\} \\
& (f \rightarrow g)(x)= \begin{cases}1 & \text { if } f(x) \leq g(x) \\
g(x) & \text { otherwise }\end{cases} \\
& (\neg f)(x)= \begin{cases}1 & \text { if } f(x)=0 \\
0 & \text { otherwise. }\end{cases}
\end{aligned}
$$

The top and bottom elements of the algebra are the constant functions 1 and 0 , respectively.

We shall denote by $\mathscr{G}(P)$ the Gödel subalgebra of the algebra of all functions from $[0,1]$ to itself generated by $P$. For each integer $k \geq 0$, we write $\mathscr{G}_{k}$ for the free Gödel algebra on $k$ free generators $X_{1}, \ldots, X_{k}$. Note that, since the variety of Gödel algebras is locally finite, $\mathscr{G}_{k}$ is finite. Since $\mathscr{G}(P)$ is generated by $n$ elements, there is a congruence $\Theta$ on $\mathscr{G}_{n}$ such that

$$
\mathscr{G}_{n} / \Theta \cong \mathscr{G}(P),
$$

where $\cong$ denotes isomorphism of Gödel algebras. Congruences of finite Gödel algebras are principal, so that $\Theta$ is generated by a single equation $\alpha\left(X_{1}, \ldots, X_{n}\right)=$ $T$ in the language of Gödel algebras. In logical terms, there is a single formula

$$
\alpha_{P} \equiv \alpha_{P}\left(X_{1}, \ldots, X_{n}\right)
$$

over the $n$ variables $X_{1}, \ldots, X_{n}$, such that the Lindenbaum algebra of the theory axiomatized by the single axiom $\alpha_{P}$ is isomorphic to $\mathscr{G}(P)$. Note that $\alpha_{P}$ is uniquely determined by $P$ up to logical equivalence. Intuitively, $\alpha_{P}$ encodes all relations between the fuzzy sets $f_{1}, \ldots, f_{n}$ that Gödel logic is capable to express.

In this section, we shall show how to obtain an explicit combinatorial representation of the algebra $\mathscr{G}(P)$ in terms of certain partially ordered sets (posets, for short). Recall that, given a poset $(F, \leq)$ and a set $Q \subseteq F$, the downset of $Q$ is

$$
\downarrow Q=\{x \in F \mid x \leq q, \text { for some } q \in Q\} .
$$

We write $\downarrow q$ for $\downarrow\{q\}$. A poset $F$ is a forest if for all $q \in F$ the downset $\downarrow q$ is a chain (i.e., a totally ordered set). A leaf is a maximal element of $F$. A tree is a forest with a bottom element, called the root of the tree. A subforest of a forest $F$ is the downset of some $Q \subseteq F$. The height of a chain is the number of its elements. The height of a forest is the height of an inclusion-maximal chain of the forest. 
Let $\operatorname{Sub}(F)$ denote the family of all subforests of a forest $F$. Notice that $\operatorname{Sub}(F)$ has a natural structure of Gödel algebra, where $\wedge$ and $\vee$ are given by union and intersection of subforests, and implication is defined, for $F_{1}, F_{2} \in$ $\operatorname{Sub}(F)$, as

$$
F_{1} \rightarrow F_{2}=\left\{q \in F \mid \downarrow q \cap F_{1} \subseteq \downarrow q \cap F_{2}\right\} .
$$

The constants $\perp, \top$ are the empty forest and $F$ itself, respectively. Finally, negation is defined by $\neg F_{1}=F_{1} \rightarrow \perp$.

We introduce a specific forest built from assignments that plays a key role in the following.

Definition 2. We say that two assignments $\mu$ and $\nu$ are equivalent over the first $n$ variables, or $n$-equivalent, written $\mu \equiv_{n} \nu$, if and only if there exists a permutation $\sigma: \underline{n} \rightarrow \underline{n}$ such that:

$$
\begin{aligned}
& 0 \preceq_{0} \mu\left(X_{\sigma(1)}\right) \preceq_{1} \cdots \preceq_{n-1} \mu\left(X_{\sigma(n)}\right) \preceq_{n} 1, \\
& 0 \preceq_{0} \nu\left(X_{\sigma(1)}\right) \preceq_{1} \cdots \preceq_{n-1} \nu\left(X_{\sigma(n)}\right) \preceq_{n} 1,
\end{aligned}
$$

where $\preceq_{i} \in\{<,=\}$, for $i=0, \ldots, n$.

Clearly, $\equiv_{n}$ is an equivalence relation. Throughout, we write $\mathscr{F}_{n}$ for the (finite) set of equivalence classes of $\equiv_{n}$.

It is not difficult to show that if $\alpha\left(X_{1}, \ldots, X_{n}\right)$ is a well-formed formula in Gödel logic, and $\mu, \nu$ are two $n$-equivalent assignments, then

$$
\mu\left(\alpha\left(X_{1}, \ldots, X_{n}\right)\right)=1 \text { if and only if } \nu\left(\alpha\left(X_{1}, \ldots, X_{n}\right)\right)=1 \text {. }
$$

We can further endow $\mathscr{F}_{n}$ with a partial order.

Definition 3. Let $[\mu]_{\equiv_{n}},[\nu]_{\equiv_{n}} \in \mathscr{F}_{n}$, and let $\sigma: \underline{n} \rightarrow \underline{n}$ be a permutation such that

$$
\begin{aligned}
& 0 \preceq_{0} \nu\left(X_{\sigma(1)}\right) \preceq_{1} \cdots \preceq_{n-1} \nu\left(X_{\sigma(n)}\right) \preceq_{n} 1, \\
& 0 \preceq_{0} \mu\left(X_{\sigma(1)}\right) \preceq_{1} \cdots \preceq_{n-1} \mu\left(X_{\sigma(n)}\right) \preceq_{n} 1,
\end{aligned}
$$

where $\preceq_{i}, \widetilde{\preceq}_{i} \in\{<,=\}$, for $i=0, \ldots, n$. We define $[\mu]_{\equiv_{n}} \leq[\nu]_{\equiv_{n}}$ if and only if there exists an index $k \in\{0, \ldots, n\}$ such that

i) $\preceq_{i}$ coincides with $\preceq_{i}$ if $0 \leq i \leq k$,

ii) $\widetilde{\preceq}_{i}$ coincides with $=$ if $k+1 \leq i \leq n$.

Example 1. Let $\mu, \nu, \xi$ be assignments such that

$-\mu\left(X_{1}\right)=1, \mu\left(X_{2}\right)=1 / 3, \mu\left(X_{3}\right)=0, \mu\left(X_{4}\right)=1$,

$-\nu\left(X_{1}\right)=1, \nu\left(X_{2}\right)=1 / 4, \nu\left(X_{3}\right)=0, \nu\left(X_{4}\right)=1 / 2$

- $\xi\left(X_{1}\right)=1, \xi\left(X_{2}\right)=1 / 2, \xi\left(X_{3}\right)=0, \xi\left(X_{4}\right)=1 / 2$.

For $\sigma(1)=3, \sigma(2)=2, \sigma(3)=4, \sigma(4)=1$, one has 
- $0=\mu\left(X_{3}\right)<\mu\left(X_{2}\right)<\mu\left(X_{4}\right)=\mu\left(X_{1}\right)=1$,

$-0=\nu\left(X_{3}\right)<\nu\left(X_{2}\right)<\nu\left(X_{4}\right)<\nu\left(X_{1}\right)=1$,

$-0=\xi\left(X_{3}\right)<\xi\left(X_{2}\right)=\xi\left(X_{4}\right)<\xi\left(X_{1}\right)=1$.

Thus, according to Definition $3,[\mu]_{\equiv_{n}} \leq[\nu]_{\equiv_{n}}$, and $[\xi]_{\equiv_{n}}$ is uncomparable to both $[\mu]_{\equiv_{n}}$ and $[\nu]_{\equiv_{n}}$.

One checks that $\leq$ in Definition 3 indeed is a partial order on $\mathscr{F}_{n}$, and $\left(\mathscr{F}_{n}, \leq\right)$ is in fact a forest $[4$, Lemma 3.3$]$. We immediately notice that

a) the roots of the trees are the classes of Boolean assignments,

b) the class $[\mu]_{\equiv_{n}}$ such that $\mu\left(X_{1}\right)=\cdots=\mu\left(X_{n}\right)=0$ is the only tree having height 1 , and

c) the leaves are those classes of assignments in which no variable is set to 1 .

For each $i=1, \ldots, n$, let $\chi_{i}=\left\{[\mu]_{\equiv_{n}} \mid \mu\left(X_{i}\right)=1\right\}$ be the $i^{\text {th }}$ generating subforest of $\mathscr{F}_{n}$.

Proposition 1. Fix an integer $k \geq 0$. (i) $\operatorname{Sub}\left(\mathscr{F}_{k}\right)$ is (isomorphic to) the free Gödel algebra on $k$ free generators. A free generating set is given by the collection of generating subforests. (ii) Up to isomorphism, the quotients of $\operatorname{Sub}\left(\mathscr{F}_{k}\right)$ are precisely the algebras of the form $\operatorname{Sub}(F)$, for $F \in \operatorname{Sub}\left(\mathscr{F}_{k}\right)$. (iii) The set of prime filters ordered by reverse inclusion of $\operatorname{Sub}(F)$ is order-isomorphic to $F$ for every $F \in \operatorname{Sub}\left(\mathscr{F}_{k}\right)$.

Proof. The proof is a straightforward translation of [5, Remark 2 and Proposition $2.4]$ in the language of assignments.

Figure 2 shows the forest $\mathscr{F}_{2}$, whose nodes are labelled by the ordering of variables under a given assignment as in (4). However, for the sake of readability, here and in the following figure we write $X_{i}$ instead of $\mu\left(X_{i}\right)$.

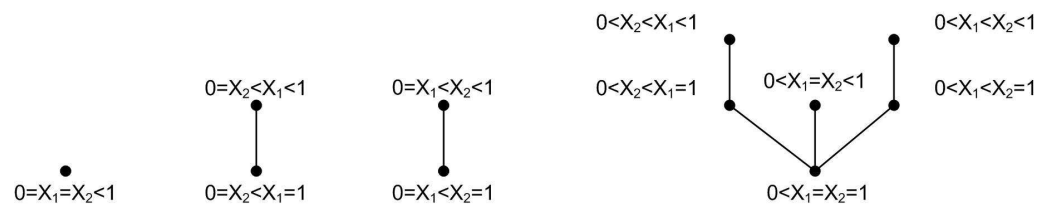

Fig. 2. The forest $\mathscr{F}_{2}$.

As an immediate consequence of Proposition 1, we can reformulate (2) as follows: $P$ uniquely determines a congruence $\Theta^{\prime}$ on $\operatorname{Sub}\left(\mathscr{F}_{n}\right)$, and a subforest $\mathscr{F}(P)$ of $\mathscr{F}_{n}$ such that

$$
\operatorname{Sub}\left(\mathscr{F}_{n}\right) / \Theta^{\prime} \cong \operatorname{Sub}(\mathscr{F}(P)) \cong \mathscr{G}(P)
$$

To relate $\Theta^{\prime}$ with the formula $\alpha_{P}$ in $(3)$ or, equivalently, with $\mathscr{F}(P)$, we shall give an explicit description of $\mathscr{F}(P)$. To this end, it is convenient to introduce the following notion. 
Definition 4. Let $[\mu]_{\equiv_{n}} \in \mathscr{F}_{n}$ and $x \in[0,1]$. We say $[\mu]_{\equiv_{n}}$ is realized by $P$ at $x$ if there exists a permutation $\sigma: \underline{n} \rightarrow \underline{n}$ such that

$$
\begin{gathered}
0 \preceq_{0} f_{\sigma(1)}(x) \preceq_{1} \cdots \preceq_{n-1} f_{\sigma(n)}(x) \preceq_{n} 1, \\
0 \preceq_{0} \mu\left(X_{\sigma(1)}\right) \preceq_{1} \cdots \preceq_{n-1} \mu\left(X_{\sigma(n)}\right) \preceq_{n} 1,
\end{gathered}
$$

where $\preceq_{i} \in\{<,=\}, i \in\{0, \ldots, n\}$.

\section{Proposition 2.}

$$
\mathscr{F}(P)=\downarrow\left\{[\mu]_{\equiv_{n}} \in \mathscr{F}_{n} \mid[\mu]_{\equiv_{n}} \text { is realized by } P \text { at somex } \in[0,1]\right\}
$$

Proof. We construct a subdirect representation of $\mathscr{G}(P)$ as follows. There exists a finite set $\left\{x_{1}, \ldots, x_{m}\right\} \subseteq[0,1]$ such that for each $y \in[0,1]$, if $[\mu]_{\equiv_{n}} \in \mathscr{F}(P)$ is realized by $P$ at $y$, then it is also realized by $P$ at $x_{i}$, for some $i \in \underline{m}$. Moreover, one checks that evaluating the elements of $\mathscr{G}(P)$ at $x_{i}$ yields a totally ordered Gödel algebra $C_{x_{i}}$ that is a homomorphic image of $\mathscr{G}(P)$ via the quotient map $q_{i}$ given by restriction to $x_{i}$. The homomorphism

$$
s: \mathscr{G}(P) \hookrightarrow \prod_{i=1}^{m} C_{x_{i}}
$$

given by

$$
g \in \mathscr{G}(P) \longmapsto\left(q_{1}(g), \ldots, q_{m}(g)\right)
$$

is injective. Indeed, let $g \neq h \in \mathscr{G}(P)$, say $g(y)>h(y)$ for $y \in[0,1]$. For the sake of brevity, we shall only deal with the case $1>g(y)>h(y)>0$. Then $g(y)=f_{i}(y)$ and $h(y)=f_{j}(y)$ for $i \neq j$. Let $[\mu]_{\equiv_{n}}$ be the assignment realized by $P$ at $y$. There exists $u \in \underline{m}$ such that $[\mu]_{\equiv_{n}}$ is realized by $P$ at $x_{u}$, and therefore $f_{i}\left(x_{u}\right)>f_{j}\left(x_{u}\right)$, which proves $s(g) \neq s(h)$.

It now follows that $s$ is a subdirect representation of $\mathscr{G}(P)$. By Proposition $1($ iii $)$ we identify prime filters of $\mathscr{G}(P)$ with elements of $\mathscr{F}(P) \subseteq \mathscr{F}_{n}$. The primes that are kernels of $q_{1}, \ldots, q_{m}$ must comprise all inclusion-minimal primes of $\mathscr{G}(P)$, i.e., all leaves of $\mathscr{F}(P)$, for otherwise $s$ could not be a subdirect representation. Therefore, the classes $[\mu]_{\equiv_{n}}$ realized by $P$ at some $x \in[0,1]$ comprise all leaves of $\mathscr{F}(P)$ (and possibly other elements). Since any forest is the downset of its leaves the proposition is proved.

Moreover, we associate with a formula $\alpha\left(X_{1}, \ldots, X_{n}\right)$ the uniquely determined subforest of $\mathscr{F}_{n}$, denoted $\mathscr{F}_{\alpha}$, as follows:

$$
\mathscr{F}_{\alpha}=\left\{[\mu]_{\equiv_{n}} \in \mathscr{F}_{n} \mid \mu(\alpha)=1\right\} .
$$

By (5), $\mathscr{F}_{\alpha}$ does not depend on the choice of $\mu$. Clearly, $\mathscr{F}_{\alpha}$ corresponds to the quotient algebra $\operatorname{Sub}\left(\mathscr{F}_{n}\right) / \Theta^{\prime}$, where $\Theta^{\prime}$ is the congruence generated by $\alpha\left(X_{1}, \ldots, X_{n}\right)=\top$. Finally, by the foregoing we have

$$
\mathscr{F}_{\alpha_{P}}=\mathscr{F}(P) \text {. }
$$




\section{Gödel Approximation of Ruspini Partitions}

Definition 5. We denote by $\mathscr{R}_{n}$ the subforest of $\mathscr{F}_{n}$ obtained by removing from $\mathscr{F}_{n}$ the single tree having height 1 , and the leaves of all the trees having height 2. We call $\mathscr{R}_{n}$ the Ruspini forest.

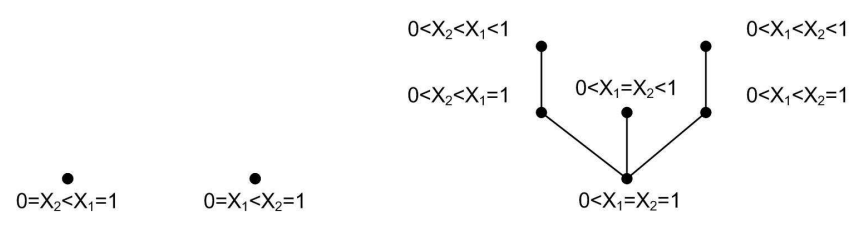

Fig. 3. The Ruspini forest $\mathscr{R}_{2}$.

We now show how to explicitly axiomatize $\mathscr{R}_{n}$.

Definition 6. We define the Ruspini axiom $\rho_{n}=\alpha \vee \beta$, where

$$
\alpha=\bigvee_{1 \leq i<j \leq n}\left(\neg \neg X_{i} \wedge \neg \neg X_{j}\right), \quad \text { and } \quad \beta=\bigvee_{1 \leq i \leq n}\left(X_{i} \wedge \bigwedge_{1 \leq j \neq i \leq n} \neg X_{j}\right) .
$$

Lemma 1. $\mathscr{F}_{\rho_{n}}=\mathscr{R}_{n}$.

Proof. Fix an assignment $\mu$. Since

$$
\mu(\neg \neg X)=\left\{\begin{array}{l}
0 \text { if } \mu(X)=0 \\
1 \text { otherwise, }
\end{array}\right.
$$

$\mu(\alpha) \neq 1$ if and only if at most one variable $X_{i_{0}}$ satisfies $\mu\left(X_{i_{0}}\right) \neq 0$.

Observe now that $\mu(\beta)=1$ if and only if there exists $i \in \underline{n}$ such that, for $j \neq i, \mu\left(X_{i}\right)=1$ and $\mu\left(X_{j}\right)=0$.

Therefore, $\mu\left(\rho_{n}\right)=\mu(\alpha \vee \beta) \neq 1$ if and only if there exists $i_{0} \in \underline{n}$ such that, for $j \neq i_{0}, \mu\left(X_{i_{0}}\right)<1$ and $\mu\left(X_{j}\right)=0$. It is now straightforward to verify that the latter condition holds if and only if $[\mu]_{\equiv_{n}} \notin \mathscr{R}_{n}$.

Let us introduce a property of $P$ that we shall use in our main result. Let $\lambda:[0,1] \rightarrow[0,1]$ be an order preserving map such that $\lambda(0)=0$ and $\lambda(1)=1$, and let $t=\inf \lambda^{-1}(1)$. If the restriction of $\lambda$ to $[0, t]$ is an order isomorphism between $[0, t]$ and $[0,1]$, we say $\lambda$ is a comparison map.

Definition 7. We say $P$ is a weak Ruspini partition if for all $x \in[0,1]$, there exist $y \in[0,1]$, a comparison map $\lambda$, and an order isomorphism $\gamma:[0,1] \rightarrow[0,1]$ such that

(i) $\lambda\left(f_{i}(y)\right)=f_{i}(x)$, for all $i \in \underline{n}$. 
(ii) $\sum_{i=1}^{n} \gamma\left(f_{i}(y)\right)=1$.

Lemma 2. Let $[\mu]_{\equiv_{n}}, \quad[\nu]_{\equiv_{n}} \in \mathscr{F}_{n}$ and $x, y \in[0,1]$ such that $[\mu]_{\equiv_{n}}$ and $[\nu]_{\equiv_{n}}$ are realized by $P$ at $x$ and $y$, respectively. Then the following are equivalent.

(i) $[\mu]_{\equiv_{n}} \leq[\nu]_{\equiv_{n}}$.

(ii) There exists a comparison map $\lambda:[0,1] \rightarrow[0,1]$ with $\lambda\left(f_{i}(y)\right)=f_{i}(x)$, for all $i \in \underline{n}$.

Moreover, the following are equivalent.

(iii) $[\mu]_{\equiv_{n}}$ is a leaf of $\mathscr{R}_{n}$.

(iv) There exists an order isomorphism $\gamma:[0,1] \rightarrow[0,1]$ with $\sum_{i=1}^{n} \gamma\left(f_{i}(x)\right)=1$.

Proof. $(i) \Rightarrow(i i)$. By Definitions 3 and 4 , there exists a permutation $\sigma: \underline{n} \rightarrow \underline{n}$ such that

$$
\begin{aligned}
& 0 \preceq_{0} f_{\sigma(1)}(y) \preceq_{1} \cdots \preceq_{n-1} f_{\sigma(n)}(y) \preceq_{n} 1, \\
& 0 \preceq_{0} f_{\sigma(1)}(x) \preceq_{1} \ldots \preceq_{n-1} f_{\sigma(n)}(x) \preceq_{n} 1,
\end{aligned}
$$

where $\preceq_{i}, \widetilde{\preceq}_{i} \in\{<,=\}$, and there is $k \in\{0, \ldots, n\}$ satisfying $i$ ) and $\left.i i\right)$ in Definition 3 . We deal with the case $k<n$ only; the case $k=n$ is a trivial variation thereof. We define $\Lambda$ by $\Lambda\left(f_{\sigma(i)}(y)\right)=f_{\sigma(i)}(x)$, for $1 \leq i \leq k$, and $\Lambda\left(f_{\sigma(i)}(y)\right)=1$ if $k+1 \leq i \leq n$. We extend $\Lambda$ to a comparison map as follows. Consider the closed intervals $I_{0}=\left[0, f_{\sigma(1)}(y)\right], J_{0}=\left[0, f_{\sigma(1)}(x)\right], I_{i}=\left[f_{\sigma(i)}(y), f_{\sigma(i+1)}(y)\right]$ and $J_{i}=\left[f_{\sigma(i)}(x), f_{\sigma(i+1)}(x)\right]$, for $1 \leq i \leq k$. Now let us fix $0 \leq h \leq k$. Note that if $I_{h}$ collapses to a point, then $J_{h}$ also collapses to a point. Therefore in all cases we can choose order isomorphisms $\lambda_{h}: I_{h} \rightarrow J_{h}$. Moreover, set $I_{k+1}=\left[f_{\sigma(k+1)}(y), 1\right]$ and $\lambda_{k+1}: I_{k+1} \rightarrow\{1\}$. Since $\lambda_{h}$ and $\lambda_{h+1}$ agree at $I_{h} \cap I_{h+1}$ by construction, the function $\lambda:[0,1] \rightarrow[0,1]$ defined by $\lambda(r)=\lambda_{j}(r)$ if $r \in I_{j}$, for $0 \leq j \leq k+1$, is a comparison map satisfying (ii).

$($ ii $) \Rightarrow(i)$. Immediate from Definitions 3 and 4.

(iii) $\Rightarrow(i v)$. It is an exercise to check that $[\mu]_{\equiv_{n}}$ is a leaf of $\mathscr{R}_{n}$ if and only if exactly one of the following two cases hold.

Case 1. There exists $i_{0}$ such that $\mu\left(X_{i_{0}}\right)=1$ and $\mu\left(X_{i}\right)=0$ for $i \neq i_{0}$.

Let $\gamma$ be the identity map. By Definition 4 , we have $\sum_{i=1}^{n} \gamma\left(f_{i}(x)\right)=1$.

Case 2. For all $i, \mu\left(X_{i}\right)<1$, and there exist $i_{0}, i_{1}$ such that $0<\mu\left(X_{i_{0}}\right) \leq \mu\left(X_{i_{1}}\right)$. Let us write

$$
0 \preceq_{0} f_{\sigma(1)}(x) \preceq_{1} \cdots \preceq_{n-1} f_{\sigma(n)}(x) \preceq_{n} 1,
$$

for some permutation $\sigma$ and $\preceq_{i} \in\{<,=\}$. We shall assume $\preceq_{0}$ is $<$. The case where some $f_{i}$ takes value zero at $x$ is entirely similar.

Now consider the $(n-1)$-dimensional simplex ${ }^{3} S_{n}$, given by the convex hull of the standard basis of $\mathbb{R}_{n}$. Let $S_{n}^{(1)}$ be the simplicial complex given by the first barycentric subdivision of $S_{n}$. The $(n-1)$-dimensional simplices of $S_{n}^{(1)}$ are in bijection with permutations of $\underline{n}$, and the solution set of the inequalities

$$
0 \leq r_{1} \leq \cdots \leq r_{n} \leq 1
$$

\footnotetext{
${ }^{3}$ For all unexplained notions in combinatorial topology, please see [8].
} 
in $S^{n}$ is an $(n-1)$-dimensional simplex $S \in S_{n}^{(1)}$. Consider the equalities

$$
r_{i}=r_{i+1}
$$

for each $i=1, \ldots, n-1$ such that $\preceq_{i}$ is $=$. Then the solution set of (7) and (8) is a nonempty face $T$ of $S$. Consider next the strict inequalities

$$
\left\{\begin{array}{l}
r_{i}<r_{i+1} \\
0<r_{1} \\
r_{n}<1
\end{array}\right.
$$

for all $i=1, \ldots, n-1$ such that $\preceq_{i}$ is $<$. Then the solution set of (7), (8), and (9) is the relative interior $T^{\circ}$ of $T$. Since $T$ is nonempty, $T^{\circ}$ is nonempty. The barycenter $b=\left(b_{1}, \ldots, b_{n}\right)$ of $T$ lies in $T^{\circ}$. Since $b \in S_{n}$, we have $\sum_{k=1}^{n} b_{k}$. Moreover, by construction,

$$
0 \preceq_{0} b_{1} \preceq_{1} \cdots \preceq_{n-1} b_{n} \preceq_{n} 1 .
$$

We define $\Gamma$ by $\Gamma\left(f_{\sigma(i)}\right)=b_{i}$. Arguing as in the proof of $(i) \Rightarrow(i i)$, we conclude that there is an extension of $\Gamma$ to an order isomorphism $\gamma:[0,1] \rightarrow[0,1]$ satisfying (iv).

$(i v) \Rightarrow(i i i)$. Suppose $[\mu]_{\equiv_{n}}$ is not a leaf of $\mathscr{R}_{n}$. Thus, exactly one of the following two cases holds.

Case 1. $[\mu]_{\equiv_{n}} \in \mathscr{F}_{n} \backslash \mathscr{R}_{n}$.

In this case there exists $i_{0}$ such that $\mu\left(X_{i_{0}}\right)<1$ and $\mu\left(X_{i}\right)=0$ for $i \neq i_{0}$. Using Definition 4, we have $\sum_{i=1}^{n} \gamma\left(f_{i}(x)\right)<1$, for each order isomorphism $\gamma$.

Case 2. $[\mu]_{\equiv_{n}} \in \mathscr{R}_{n}$, but $[\mu]_{\equiv_{n}} \in \mathscr{R}_{n}$ is not a leaf of $\mathscr{R}_{n}$.

It is easy to check that there exist $i_{0}, i_{1}$ such that $0<\mu\left(X_{i_{0}}\right) \leq \mu\left(X_{i_{1}}\right)=1$. Using Definition 4, we have $f_{i_{1}}(x)=1$ and $f_{i_{0}}(x)>0$, and thus $\sum_{i=1}^{n} \gamma\left(f_{i}(x)\right)>$ 1 , for each order isomorphism $\gamma$.

To state our main result we still need to show how to obtain a formula $\psi_{[\mu]_{\equiv_{n}}}$ associated with a given element $[\mu]_{\equiv_{n}} \in \mathscr{F}_{n}$ such that $\psi_{[\mu]_{\equiv_{n}}}$ evaluates to 1 exactly on $\downarrow[\mu]_{\equiv_{n}}$. For this, we define the derived connective $\alpha \triangleleft \beta=((\beta \rightarrow$ $\alpha) \rightarrow \beta$ ). Given an assignement $\mu$ we have that

$$
\mu(\alpha \triangleleft \beta)= \begin{cases}1 & \text { if } \mu(\alpha)<\mu(\beta) \text { or } \mu(\alpha)=\mu(\beta)=1 \\ \mu(\beta) & \text { otherwise. }\end{cases}
$$

Suppose now that, for a given permutation $\sigma: \underline{n} \rightarrow \underline{n}$,

$$
0 \preceq_{0} \mu\left(X_{\sigma(1)}\right) \preceq_{1} \cdots \preceq_{n-1} \mu\left(X_{\sigma(n)}\right) \preceq_{n} 1,
$$

where $\preceq_{i} \in\{<,=\}, i=0, \ldots, n$. We associate to $[\mu]_{\equiv_{n}}$ the formula

$$
\psi_{[\mu]_{\equiv_{n}}}=\left(\perp \bowtie_{0} X_{\sigma(1)}\right) \wedge\left(X_{\sigma(1)} \bowtie_{1} X_{\sigma(2)}\right) \wedge \cdots \wedge\left(X_{\sigma(n)} \bowtie_{n} \top\right),
$$

where $\bowtie_{i}=\triangleleft$ if $\preceq_{i}$ is $<$, and $\bowtie_{i}=\leftrightarrow$ otherwise. 
Lemma 3. $\mathscr{F}_{[\mu] \equiv_{n}}=\downarrow[\mu]_{\equiv_{n}}$

Proof. We omit the straightforward verification. Compare [3,1] where a fullfledged theory of normal forms is developed.

Given a forest $F \subseteq \mathscr{F}_{n}$ let us indicate with $\operatorname{Root}(F)$ the set of roots of $F$ (i.e. the classes of Boolean assignments over the first $n$ variables). If $r \in \operatorname{Root}(F)$, we write Leaf $(r, F)$ for the set of leaves of $F$ above the root $r$.

Definition 8. We say that a forest $F$ is a Ruspini subforest if $F \subseteq \mathscr{R}_{n}$ and each leaf of $F$ is a leaf of $\mathscr{R}_{n}$.

We write $\vdash \alpha$ if Gödel logic proves the formula $\alpha$; equivalently, by completeness, if $\mu(\alpha)=1$ for all assignments $\mu$. We can finally prove our main result.

Theorem. The following are equivalent.

(i) $P$ is a weak Ruspini partition.

(ii) $\mathscr{F}(P)$ is a Ruspini subforest.

(iii) $\vdash \alpha \wedge \beta \wedge \gamma$, where

$\alpha=\left(\alpha_{P} \rightarrow \rho_{n}\right)$,

$\beta=\bigwedge_{r \in \operatorname{Root}\left(\mathscr{R}_{n}\right)} \bigwedge_{l \in \operatorname{Leaf}\left(r, \mathscr{R}_{n}\right)}\left(\left(\psi_{l} \rightarrow \alpha_{P}\right) \vee\left(\left(\psi_{l} \wedge \alpha_{P}\right) \rightarrow \psi_{r}\right)\right)$,

$\gamma=\bigwedge_{r \in \operatorname{Root}\left(\mathscr{R}_{n}\right)}\left(\left(\psi_{r} \rightarrow \alpha_{P}\right) \rightarrow\left(\bigvee_{l \in \operatorname{Leaf}\left(r, \mathscr{R}_{n}\right)}\left(\psi_{l} \rightarrow \alpha_{P}\right)\right)\right)$.

Moreover, for any Ruspini subforest $F$ there exists a Ruspini partition $P^{\prime}=$ $\left\{f_{1}^{\prime}, \ldots, f_{n}^{\prime}\right\}$, with $f_{i}^{\prime}:[0,1] \rightarrow[0,1]$, such that $\mathscr{F}\left(P^{\prime}\right)=F$.

Proof. $(i) \Rightarrow($ ii $)$. By Lemma 2, we can reformulate Definition 7 in terms of assignments as follows. For all $[\mu]_{\equiv_{n}} \in \mathscr{F}_{n}$ realized by $P$ at some $x \in[0,1]$, there exists $[\nu]_{\equiv_{n}} \geq[\mu]_{\equiv_{n}}$ realized by $P$ at some $y \in[0,1]$ such that $[\nu]_{\equiv_{n}}$ is a leaf of $\mathscr{R}_{n}$. Thus, by Proposition 2, $\mathscr{F}(P)$ is exactly the downset of those leaves of $\mathscr{R}_{n}$ realized by $P$ at some $x \in[0,1]$.

(ii) $\Rightarrow$ (iii). Let $r \in \operatorname{Root}\left(\mathscr{R}_{n}\right)$. If $r \notin \mathscr{F}(P)$ then the set Leaf $(r, \mathscr{F}(P))$ is empty, and by (6) the formula $\alpha_{P}$ evaluates to zero under all assignments $\mu$ such that $[\mu]_{\equiv_{n}} \geq r$. Thus, for all $l \in \operatorname{Leaf}\left(r, \mathscr{R}_{n}\right),\left(\psi_{l} \wedge \alpha_{P}\right)$ is a contradiction and $\left(\psi_{l} \wedge \alpha_{P}\right) \rightarrow \psi_{r}$ is a tautology. Therefore, the conjucts of $\beta$ indexed by $r \notin \mathscr{F}(P)$ are tautologies. Moreover, since $\psi_{r}$ and $\psi_{l}$ evaluate to zero under all assignments $\mu$ such that $[\mu]_{\equiv_{n}} \nsucceq r$, and to a value different from zero otherwise, $\left(\psi_{r} \rightarrow \alpha_{P}\right) \leftrightarrow\left(\psi_{l} \rightarrow \alpha_{P}\right)$ is a tautology for all $l \in \operatorname{Leaf}\left(r, \mathscr{R}_{n}\right)$, and then the conjucts of $\gamma$ indexed by $r \notin \mathscr{F}(P)$ are tautologies. Let now $r \in \mathscr{F}(P)$, and let $l \in \operatorname{Leaf}\left(r, \mathscr{R}_{n}\right)$. If $l \in \mathscr{F}(P)$ then $\psi_{l} \rightarrow \alpha_{P}$ is a tautology, otherwise $\left(\psi_{l} \wedge \alpha_{P}\right) \rightarrow \psi_{r}$ is a tautology. Thus, every formula in $\beta$ indexed by $r \in \mathscr{F}(P)$ is a tautology. Moreover, $\operatorname{Leaf}(r, \mathscr{F}(P)) \neq \emptyset$, and for $l_{0} \in \operatorname{Leaf}(r, \mathscr{F}(P)), \psi_{l_{0}} \rightarrow \alpha_{P}$ is a tautology. Therefore, every formula in $\gamma$ indexed by $r \in \mathscr{F}(P)$ is a tautology. We thus obtain that $\beta$ and $\gamma$ are tautologies. Since $\alpha$ also is a tautology by the hypothesis $\mathscr{F}(P) \subseteq \mathscr{R}_{n}$, we obtain that whenever (ii) holds, $\alpha \wedge \beta \wedge \gamma$ is a tautology. 
(iii) $\Rightarrow($ i). Suppose $P$ is not a weak Ruspini partition. By Definition 7 , using Lemma 2 and $(6)$, there exists $[\mu]_{\equiv_{n}} \in \mathscr{F}_{\alpha_{P}}$ such that exactly one of the following two condition hold.

(a) $[\mu]_{\equiv_{n}} \in \mathscr{F}_{n} \backslash \mathscr{R}_{n}$.

(b) $[\mu]_{\equiv_{n}} \in \mathscr{R}_{n}$ is a maximal element of $\mathscr{F}_{\alpha_{P}}$, but it is not a leaf of $\mathscr{R}_{n}$.

If ( $a$ ) holds then, clearly, $\mu(\alpha) \neq 1$. As to $(b)$, let $r \leq[\mu]_{\equiv_{n}}$ be a root of $\mathscr{F}_{n}$. If $r=[\mu]_{\equiv_{n}}$, then the formula $\psi_{r} \rightarrow \alpha_{P}$ is a tautology, while $\psi_{l} \rightarrow \alpha_{P}$ does not evaluate to 1 at $l$, for all $l \in \operatorname{Leaf}\left(r, \mathscr{R}_{n}\right)$. Thus, $\gamma$ is not a tautology. If $r \neq[\mu]_{\equiv_{n}}$ then for all $l \in \operatorname{Leaf}\left(r, \mathscr{R}_{n}\right), l \geq[\mu]_{\equiv_{n}}, \psi_{l} \rightarrow \alpha_{P}$ and $\left(\psi_{l} \wedge \alpha_{P}\right) \rightarrow \psi_{r}$ evaluate to zero at $l$. Therefore $\beta$ is not a tautology. In any case, $\alpha \wedge \beta \wedge \gamma$ is not a tautology.

Finally, we prove the last statement of the theorem. Let $\left[\mu_{1}\right]_{\equiv_{n}}, \ldots,\left[\mu_{m}\right]_{\equiv_{n}}$ be the leaves of $F$. Partition the interval $[0,1]$ into $m$ intervals $I_{1}=\left[0, x_{1}\right]$, $I_{2}=\left(x_{1}, x_{2}\right], \ldots, I_{m}=\left(x_{m-1}, 1=x_{m}\right]$. We construct the functions $f_{i}^{\prime}$ as follows. For $i \in \underline{n}, j \in \underline{m}$, we set $f_{i}^{\prime}(x)=C_{i j} \in \mathbb{R}$ if $x \in I_{j}$. The constants $C_{i j}$ are chosen so that

(a) $\left[\mu_{j}\right]_{\equiv_{n}}$ is realized by $P^{\prime}$ at $x_{j}$,

(b) $\sum_{i=1}^{n} C_{i j}=1$.

Obviously, it is always possible to choose $C_{i j}$ so that $(a)$ holds. The proof of $($ iii $) \Rightarrow($ iv $)$ in Lemma 2 shows that, in fact, it is always possible to choose $C_{i j}$ so that both $(a)$ and $(b)$ hold.

In $\left[2\right.$, Theorem 3] it is shown that the number of leaves of $\mathscr{F}_{n}$ is

$$
L_{n}=2 \sum_{k=1}^{n} k !\left\{\begin{array}{l}
n \\
k
\end{array}\right\}
$$

where $\left\{\begin{array}{l}n \\ k\end{array}\right\}$ is the number of partitions of an $n$-element set into $k$ classes, i.e. the Stirling number of the second kind. The number $\sum_{k=1}^{n} k !\left\{\begin{array}{l}n \\ k\end{array}\right\}$ is the $n^{\text {th }}$ ordered Bell number, i.e. the number of all ordered partitions of $\underline{n}$. Compare sequence $A 000670$ in [10].

Consider $P^{\prime}=\left\{f_{1}^{\prime}, \ldots, f_{n}^{\prime}\right\}$, where $f_{i}^{\prime}:[0,1] \rightarrow[0,1]$. In the light of Section 2, let us say that $P^{\prime}$ is Gödel-equivalent to $P$ if $\mathscr{F}(P)=\mathscr{F}\left(P^{\prime}\right)$, or, equivalently, $\vdash \alpha_{P} \leftrightarrow \alpha_{P^{\prime}}$. Then:

Corollary 1. The number of classes of Gödel-equivalent weak Ruspini partitions of $n$ elements is $2^{L_{n}-1}-1$, where $L_{n}$ is given by (10).

Proof. A weak Ruspini partition $P$ is characterized, up to Gödel-equivalence, by the forest $\mathscr{F}(P)$, and therefore by a subset of leaves of $\mathscr{R}_{n}$. Noting that the number of leaves of $\mathscr{R}_{n}$ is $L_{n}-1$, and that for every weak Ruspini partition $P$, $\mathscr{F}(P) \neq \emptyset$, the corollary follows.

Corollary 2. (i) There is a Ruspini subforest $F$ such that whenever $\mathscr{F}(P)=F$ then each $f_{i} \in P$ has a point of discontinuity. (ii) For all Ruspini subforests $F$ 
with $L$ leaves there is a choice of a Ruspini partition $P^{\prime}=\left\{f_{1}^{\prime}, \ldots, f_{n}^{\prime}\right\}$, with $\mathscr{F}\left(P^{\prime}\right)=F$ such that each $f_{i}^{\prime}:[0,1] \rightarrow[0,1]$ has at most $L-1$ points of discontinuity.

Proof. ( $i$ ) It suffices to choose $F \subseteq \mathscr{R}_{n}$ as the forest of all Boolean assignments which are leaves of $\mathscr{R}_{n}$. (ii) The construction used in the proof of the last statement of Theorem 3 yields the desired $P^{\prime}$.

\section{Conclusions}

Our analysis shows that Gödel logic does not have sufficient expressive power to capture the Ruspini condition (1). However, we have proved that Gödel logic does capture the notion of weak Ruspini partition in Definition 7. Moreover, our Theorem 3 shows that weak Ruspini partitions indeed are the best available approximation of Ruspini partitions in Gödel logic: for each weak Ruspini partition $P$, there exists a Ruspini partition $P^{\prime}$ that is Gödel-equivalent to $P$. Thus, there is no formula in Gödel logic telling $P$ and $P^{\prime}$ apart. Moreover, Corollary 2 shows that one can always choose a Ruspini partition $P^{\prime}$ whose elements have a bounded number of points of discontinuity. Finally, up to Gödel equivalence, there is a finite number of weak Ruspini partitions of $n$ elements, and Corollary 1 gives an exact formula to compute this number.

\section{References}

1. Aguzzoli, S., D'Antona, O.M., Marra, V.: Algorithms in propositional Gödel logic. In preparation.

2. Aguzzoli, S., Gerla, B., Manara, C.: Poset representation for Gödel and Nilpotent Minimum logics. In: ECSQARU 2005, Proceedings. Volume 3571 of Lecture Notes in Computer Science., Springer (2005) 662-674

3. Baaz, M., Veith, H.: Interpolation in fuzzy logic. Arch. Math. Logic 38(7) (1999) 461-489

4. Codara, P., D'Antona, O.M., Marra, V.: Propositional Gödel logic and Delannoy paths. In: Fuzz-IEEE 2007, Proceedings. (2007) To appear.

5. D'Antona, O.M., Marra, V.: Computing coproducts of finitely presented Gödel algebras. Ann. Pure Appl. Logic 142(1-3) (2006) 202-211

6. Gottwald, S.: A treatise on many-valued logics. Volume 9 of Studies in Logic and Computation. Research Studies Press Ltd., Baldock (2001)

7. Hájek, P.: Metamathematics of fuzzy logic. Volume 4 of Trends in Logic-Studia Logica Library. Kluwer Academic Publishers, Dordrecht (1998)

8. Rourke, C.P., Sanderson, B.J.: Introduction to piecewise-linear topology. Springer Study Edition. Springer-Verlag, Berlin (1982) Reprint.

9. Ruspini, E.H.: A new approach to clustering. Information and Control 15 (1969) 22-32

10. Sloane, N.J.A.: The on-line encyclopedia of integer sequences. Published electronically at http://www.research.att.com/ njas/sequences/ (2006) 